\title{
The Use of AZT to Reduce the Risk of Vertical Transmission of Human Immunodeficiency Virus
}

\author{
V. Bhargavi Rao, B.A. ${ }^{* \dagger}$
}

\section{INTRODUCTION}

In 1981, when the first cases of Acquired Immune Deficiency Syndrome (AIDS) were being reported and documented, AIDS was believed to occur solely amongst homosexual men. This shaped public opinion of the disease, and so Human Immunodeficiency Virus (HIV) infection was labeled as a "gay plague", excluding women and children as potential victims. Shortly thereafter, however, cases of infected female intravenous drug users surfaced and the first case of pediatric AIDS was soon identified in 1983 (1).

The World Health Organization (WHO) estimates that, since the beginning of the AIDS epidemic, approximately 13 million men, women and children have been infected with HIV. Moreover, Banatvala et al. (2) calculate that there are currently five million seropositive women of childbearing age world-wide. Another study (3) suggests that in the USA, up to $18 \%$ of all AIDS cases are women, $75 \%$ of whom are of childbearing age. In some parts of Sub-Saharan Africa, up to $30 \%$ of all pregnant women are HIV-positive (4). It is obvious that, contrary to earlier assumptions, AIDS is far from being a "gay plague", and that heterosexual women are now a significant proportion of those infected. This has profound implications for the issue of mother-to-child transmission.

Vertical transmission is said to cause roughly $90 \%$ of all pediatric HIV infection (5). Pediatric AIDS already accounts for up to $2 \%$ of all AIDS cases (6) and children born to HIV-positive women are the fastest growing population of HIV-infected patients (7). The WHO estimates that there are more than two million HIV-

\footnotetext{
* To whom correspondence should be addressed: University College, Oxford, UK OX14BH, UK; bhargavi.rao@univ.ox.ac.uk.

$\dagger$ Oxford Medical School, Oxford University, Oxford, UK.
}

infected children world-wide and that this will rise to 10 million by the year 2000 , i.e., $25 \%$ of the overall projected total (8). AIDS is now thought to be amongst the 10 leading causes of death in children aged under five years, and will soon be the fifth largest killer among children of all ages (7).

Rates of vertical transmission of HIV-1 vary geographically ranging from 14 to $40 \%$ (8). It is important to make allowances for the diagnostic criteria used in the various estimates. The vertical transmission rate of HIV-1 may well be more similar world-wide, although the potential importance of other factors that could be the basis for this diversity, such as different strains of the virus or stages of the maternal disease, should not be underestimated.

Set against this background, the Pediatric AIDS Clinical Trial Group 076 (ACTG076), in November 1994 (9), showed that in women with mildly symptomatic HIV disease and no prior treatment with antiretroviral drugs, the administration of Zidovudine (AZT; see Box 1) antepartum, intrapartum (during the delivery) and to the infant six weeks after delivery, reduced the relative risk of vertical transmission of HIV by $67.5 \%$ - a huge step forward for the treatment of HIV-infected pregnant women. This prompted rapid legislation, in many Western countries, making this treatment available to all women in this category.

This article addresses various issues raised by the ACTG076 trial. The following objectives are considered: (i) to highlight the global significance of mother-to-child transmission of HIV-1; (ii) to consider and evaluate the dramatic findings of the ACTG076 trial; (iii) to put forward ideas and discuss how AZT may be effective in reducing the risk of vertical transmission; and (iv) to provoke thought and debate on the implications of the ACTG076 trial results and the way forward. 
Table 1. Results of the ACTG076 trial.

\begin{tabular}{lcc}
\hline \hline & AZT & Placebo \\
\hline Number of infants enrolled & 180 & 183 \\
Number of HIV-Positive infants & 13 & 40 \\
$\%$ transmission $^{\mathrm{a}}$ & 8.3 & 25.5 \\
\hline \hline
\end{tabular}

PEDIATRIC AIDS CLINICAL TRIAL GROUP PROTOCOL 076 (ACTG076)

The ACTG076 trial was designed to determine the efficacy, safety and tolerance of AZT in the prevention of transmission of HIV from mother to child. As mentioned previously, AZT was administered antepartum, intrapartum and to the infant for six weeks following birth. At the first interim analysis of efficacy, the Data and Safety Monitoring Board recommended that enrollment of additional patients be discontinued and that all patients receiving the placebo in a blinded fashion be offered AZT treatment (9-11).

ACTG076 was a multi-centre, Phase III, double blind, placebo-controlled, randomized clinical trial set up to evaluate whether AZT administered to HIV-infected pregnant women, (between 14 to 36 weeks gestation and CD4 T-lymphocyte counts > 200 cells $/ \mathrm{mm}^{3}$ ) and their infants could reduce the rate of transmission from mother to infant.

Of the 477 eligible women, 409 gave birth during the trial period to a total of 415 live infants. Only 363 infants had sufficient data to be included in the analysis, 180 in the AZT group and 183 in the placebo group (Table 1).

The regimen of AZT administered to the mother during pregnancy, labor and delivery, as well as to her infant in the first six weeks of life, significantly reduced the risk of materno-fetal transmission of HIV from $25.5 \%$ to $8.3 \%$ (for women with baseline CD4 lymphocyte counts > 200 cells $/ \mathrm{mm}^{3}$ ). The difference in Kaplan-Meier percentages (i.e., the risk reduction) was highly significant ( $p=$ 0.00006). If the absolute risk reduction is $17.2 \%$, then it can be calculated that only six women would need to be treated to prevent one infant infection. Thus, it was concluded that the results of the ACTG076 correspond to a $67.5 \%$ reduction in the relative risk of transmission.

\section{QUESTIONS ARISING FROM THE ACTG076 TRIAL}

The ACTG076 trial was undeniably a milestone in the treatment of HIV-positive pregnant women. However, before the knowledge gained can be put into widespread practice, there are several fundamental questions that arise from the trial, which must be answered.

Materno-fetal transmission can occur in utero, intrapartum and postpartum. The ACTG076 trial covered all possibilities by administering AZT throughout pregnancy, except in the first trimester, and so the relative individual contributions of each period at risk were not assessed. The trial did not address the efficacy of therapy started after 34 weeks of gestation or the consequences of AZT treatment in the first trimester. Thus, the results of the ACTG076 trial cannot be easily applied to the needs of women in developing countries where a long period of AZT administration is economically unfeasible and antenatal care is for the privileged few.

It is uncertain whether the regimen used in Protocol 076 would be as potent in women with clinical characteristics differing from those enrolled in the trial. AZT may be less effective in women with advanced disease (whose infants are known to have a higher risk of becoming infected) or with low CD4 counts (3). Women in geographic areas with lower vertical transmission rates than those included in the study may not exhibit the same dramatic result. It is unknown whether AZT during pregnancy would still be expected to have an effect in women who have been treated with AZT prior to pregnancy, and who may harbor AZT resistant strains.

Although the trial results indicate that the short-term toxicity of the AZT regimen was minimal and reversible, the long-term effects on both mother and infant are unknown. Larger numbers of exposed infants need to be monitored for prolonged periods to clarify the issue of short and long-term toxicity.

An easily overlooked fact is that some infants were infected despite AZT treatment. A number of reasons, such as viral transmission before the start of treatment, non-compliance with the regimen, insufficient suppression of maternal viral replication by AZT, or some unique characteristics of the infecting strain, may explain this. If a strategy to effectively reduce the vertical transmission of HIV is to be formulated, all these questions must be addressed.

Unfortunately, very few investigations have been done to elucidate this situation. Studies by Boyer (12) and Matheson (13) confirmed that vertical transmission was reduced with antenatal AZT therapy, and also showed that the benefits may extend to women with lower CD4 counts. However, both these experiments were observational and non-randomized, therefore limited in their ability to assess drug efficacy. In addition, trials across America and Puerto Rico (14-16), Spain (17) and Argentina (18) have replicated the ACTG076 findings but have not pushed forward the frontiers of knowledge, either due to practical or ethical concerns.

Perhaps the most fundamental question that has yet to be answered is the actual mechanism by which AZT works to reduce the risk of materno-fetal transmission, 


\section{Box 1. Summary of AZT therapy}

Also known as 3'-azido-3'-deoxythymidine, zidovudine (ZDV) or retrovir commercially, AZT is a structural analogue of thymidine and is phosphorylated by cellular enzymes to an endpoint of AZT-triphosphate (AZTTP). AZTTP competes with equivalent triphosphates in the formation of proviral DNA by the viral reverse transcriptase, i.e., a nucleoside reverse transcriptase inhibitor. When AZTTP is incorporated in the nascent viral DNA strand, it results in chain termination and, hence, blocks viral replication. This action is about 100 times more specific for viral enzymes than for human enzymes.

The timing of the start of therapy remains controversial, dependent on the clinical picture as well as the viral load and CD4 count of the patient. However, trials demonstrate that AZT therapy, in asymptomatic or mildly symptomatic patient with impaired immunity, does delay the progression to AIDS or AIDS Related Complex. Low dose AZT therapy $(500 \mathrm{mg} / \mathrm{d})$ appears to be as effective as high doses $(1200 \mathrm{mg} / \mathrm{d})$ but with less side effects. The minimum efficacious dose has not yet been defined.
Previous fears of resistance to AZT monotherapy have been partially laid to rest with the advent of combination therapy, e.g., nucleoside reverse transcriptase inhibitors (didanosine, ddI; lamivudine, 3TC; zalcitabine, ddC) or protease inhibitors (ritonavir, saquinavir, indinavir). However, whilst AZT is the mainstay of treatment of HIV infection in developed countries and despite the advances made in recent years, it remains economically inaccessible for the many millions infected in the Third World.

\section{FOR REVIEW SEE:}

Doran, CM. New approaches to using antiretroviral therapy for the management of HIV infection. Annals of Pharmacotherapy 31: 228-236; 1997

Gazzard, B. What we know so far. AIDS 10 (Supplement 1): S3-7; 1996

Lange, JM. Changing therapy in HIV. AIDS 10 (Supplement 1): S27-30; 1996.

British National Formulary March 33: 270-273; 1997.

Rachlis, AR. AZT (Retrovir) update. Canadian Medical Association Journal 143: $1177-1185 ; 1990$. and it is this question on which discussion will now focus.

\section{AZT IN PREGNANCY}

The goal of AZT antiretroviral therapy during pregnancy is to maintain maternal health and reduce the risk of vertical viral transmission throughout gestation and delivery, while keeping toxicity risks low. The question as to whether to routinely treat HIV-positive pregnant women is confounded by the fact that only approximately $30 \%$ of children born to HIV-infected mothers are documented as infected within the first few years of life (8). Many uninfected and presumably healthy fetuses might be exposed to maternally administered drugs. Examination of the handling of AZT in pregnant women should aid in the prediction of efficacy and toxicity of the drug. However, most of the studies performed have been beset with the difficulty of finding a suitable model or accumulating sufficient numbers of pregnant women to assess the interaction of pregnancy and AZT.

\section{Pharmacokinetics of AZT in Pregnancy}

Studies by Watts et al. (19) and O'Sullivan et al. (20) in pregnant women, albeit using small numbers, found that the pharmacokinetics of AZT are not altered by the state of pregnancy.

\section{Transplacental Passage of AZT}

Of the AZT transferred across the placenta in an in vitro perfused model, over $90 \%$ of it remained unchanged (21). In the follow-up paper (22), the authors stated that the $10 \%$ of AZT retained was not only extensively metabolized, but that one of the metabolites was AZT-triphosphate (AZTTP) which is responsible for the antiviral activity of AZT (see Box 1). The placental content of AZTTP may reduce the potential of HIV within the placenta of infected mothers and decrease the risk of vertical transmission. In vivo studies by Pons et al. (23) and Gillet et al. (24) indicate that oral doses of AZT not only cross the placenta passively, but also set up a therapeutic concentration in the human fetus.

\section{The Placenta and AZT}

Continued fetal growth and pregnancy maintenance require a properly functioning placenta, meaning that drug effects on the placenta may have significant deleterious consequences for the fetus. The most informative experiments investigating the effects of AZT on placenta were by Bui and colleagues, using human term placenta (25). Two major cell types of interest were identified in the placenta - trophoblast cells and Hofbauer cells. Trophoblasts are capable of producing progesterone, human chorionic gonadotrophin (hCG) and human placental lactogen (hPL). The function of Hofbauer cells is not yet fully defined, but they express HLA-DR (the MHC II antigen present in mature macrophages) and CD4 (the receptor for the HIV protein gp120). 
The effect of AZT on the secretion of hPL and hCG by trophoblasts was minimal at or below a concentration of $60 \mu \mathrm{M}$. Since current therapy, at an oral dose of $100 \mathrm{mg}$ AZT every four hours, can achieve concentrations within the body of only 2 to $5 \mu \mathrm{M}$, it is unlikely that AZT will affect these hormones clinically. In comparison, progesterone production was significantly inhibited in trophoblasts at an $\mathrm{IC}_{50}$ of $3.65 \pm 0.29 \mu \mathrm{M}$, and complete inhibition seen at AZT concentrations of $20 \mu \mathrm{M}$. This marked decrease in progesterone production by AZT may have important implications for the developing fetus.

Whether placentas in the first and second trimester exhibit similar drug sensitivity remains to be determined, but as early gestational trophoblasts would exhibit a higher growth rate, the effects of AZT are likely to be enhanced. It is clear from these studies that AZT has a significant effect on placental cells in vitro, namely reduction of progesterone secretion. The next step is to see if these results can be extrapolated to gain a deeper understanding of the effects of AZT for safe clinical use in pregnant women.

\section{PROPOSED MECHANISMS BY WHICH AZT REDUCES MATERNO-FETAL TRANSMISSION}

In the article (9) first reporting the ACTG076 trial to the scientific world, Connor et al. admit that, "The mechanism by which zidovudine reduced the risk of maternal-infant transmission is not established. Maternal zidovudine treatment may have reduced the viral load and diminished the viral exposure of the fetus in utero, of the infant at delivery, or both." Four years have passed with no advance in the understanding of the mechanisms by which AZT is effective in reducing the risk of vertical transmission, despite the fact that this could be of huge potential benefit for HIV-infected pregnant women. Once the underlying mechanism has been elucidated, it follows that the treatment itself could be tailored to particular needs, situations and circumstances. Without this information, it is impossible to apply the results of the ACTG076 trial to their maximum advantage. Thus, this article will now introduce some possibilities as to how AZT is effective in this situation.

First, it is necessary to establish that HIV can be transmitted in utero, intrapartum, and postpartum. In utero infection is thought to occur in up to $50 \%$ of all vertically acquired infections, and is characterized by the detection of HIV within the neonatal period and a rapid onset of AIDS symptoms within the first few months of life. Evidence for this includes the detection of HIV in placental and fetal tissues as early as the eighth week of gestation, and isolation of HIV from amniotic fluid cells in the first and second trimester (26).
Intrapartum infection is defined by the inability to detect HIV in the early neonatal period, and the late onset of HIV-related symptoms. This is thought to occur in 50 to $70 \%$ of all cases, via exposure to maternal blood and cervicovaginal secretions in the birth canal at delivery (26).

Postpartum transmission, i.e., through breastfeeding, has been an area of uncertainty. Two European studies $(27,28)$ showed that in a cohort of pregnant HIV-positive women, those who breastfed their babies were at an increased risk of transmitting infection to their babies compared to non-breastfeeding mothers. A meta-analysis by Dunn et al. (29) calculated the attributable risk of breastfeeding at approximately $14 \%$.

Proposed determinants of materno-fetal transmission include high maternal viral load, low CD4 count, integrity of the placenta, and obstetric factors such as the duration of ruptured membranes, mode of delivery and invasive monitoring $(26,30)$. Despite the knowledge highlighted above, it is still not understood how exactly HIV-1 is transmitted between mother and child and which components are involved. It may be, for example, an opportunistic process via placental tears or ingestion of infected blood and mucus at birth, or it may be an active infecting process involving infection of the placenta and then of the fetus.

Thus, with only limited information actually available, it can be proposed that there are three main areas to classify the mechanisms by which AZT may be operating to reduce the risk of vertical transmission: (i) within the mother, (ii) within the placenta, (iii) within the fetus.

\section{Within the Mother}

In the ACTG076 trial, it was postulated that a reduction of maternal virus level was the mechanism by which AZT reduced the incidence of vertical transmission. Researchers at the European Collaborative Study (27) found that vertical transmission of HIV-1 can be correlated with the maternal viral burden and the degree of maternal immune deficiency (represented by maternal p24 antigenemia or a CD4 count less than 700). Other data suggest that HIV-1 can be transmitted from mother to infant through either cell-associated or cell-free virus (31).

More recently, the follow up study from ACTG076 trial using blood samples from the original participants (32), and a British three year trial investigating the effects of AZT therapy on vertical transmission (33), have confirmed that high maternal viral load and HIV RNA level are risk factors for vertical transmission. Sperling et al. (32) postulate, therefore, that the results of the ACTG076 could be, in part, mediated through the reduction in plasma levels of viral RNA brought about by 
AZT. O'Shea et al. (33), in their study of 95 HIV-positive women, calculated that the predicted rate of transmission relative to maternal HIV RNA was $2 \%$ at 1000 copies, $11 \%$ at 10000 copies and $40 \%$ at 100000 copies.

The next question that arises is how a reduction in viral burden is effective in preventing materno-fetal transmission. It may be that just a decrease in circulating virus, cell-associated or not (although it is not known whether both are affected by AZT therapy) is sufficient to stop infection of the fetus.

One possible theory is that intrapartum transmission occurs by exposure to the virus while the infant is in the birth canal. Studies in non-menstruating HIV-positive women find cervicovaginal secretions to contain both cell-associated and cell-free virus (34), and reports indicate that the presence and amount of HIV in genital secretions may be higher in women who are pregnant than in those who are not (35). The most provocative evidence linking this to vertical transmission comes from twin studies. A first born twin is significantly more likely to be infected than the second born, presumably related to prolonged exposure to infected blood and secretions in the genital tract prior to delivery $(32,34)$. Ho et al. (35) demonstrated that patients treated with AZT presented with a decreased viral load, both in plasma, semen and cervical fluid. Henin (36) extended this idea, correlating the excretion of free virus in cervicovaginal secretions with disease stage and AZT treatment.

It is conceivable that AZT administered to an HIVpositive mother may have an effect within the mother herself, so that certain factors apparently linked with materno-fetal transmission become less potent, therefore reducing the likelihood of the fetus becoming infected. It seems a plausible idea that, if the levels of virus are reduced, there is less virus present with the ability to infect the fetus, and currently this is probably the most widely accepted hypothesis.

\section{In the Placenta}

The placenta provides a potential barrier between maternal and fetal circulation. During the nine months of gestation, the developing fetus is separated from the infected maternal blood within the placenta by the villous wall. The mechanisms by which HIV penetrates the placental barrier are not clear. Some possibilities include passive migration of HIV into the fetal circulation through pathological lesions in the trophoblast layers which occur throughout gestation and delivery (37), cell-mediated routes of infection via Hofbauer cells or trophoblasts $(38,39)$, or even by active antibody-virus complex transmission, whereby virus bound to $\mathrm{IgG}$ is selectively taken up by binding to the Fc fragment expressed by placental cells. If placental infection with HIV is associated with vertical transmission, then AZT during pregnancy could potentially prevent placental infection and/or restrict HIV replication within cells.

It has already been shown that AZT administered to pregnant women is passively transferred across the placenta essentially unchanged (21). Of the AZT retained within the placenta, over $50 \%$ is metabolized (22). Metabolites include AZTTP, which interferes with HIV replication through inhibition of viral reverse transcriptase and so inhibits proviral DNA synthesis, and AZT-monophosphate (AZTMP), which also plays a role in inhibiting HIV replication (40).

Furman et al. (40) reported that at a clinically comparable extracellular AZT concentration of $5 \mu \mathrm{M}$, the intracellular content of AZTTP is approximately 7.5 fold higher than that required to inhibit HIV reverse transcriptase. Thus, providing the in vitro situation applies in vivo, the present dosing regimen should be adequate to produce inhibitory concentrations in both trophoblasts and Hofbauer cells.

The placenta is an important potential portal of infection for the fetus, although the mechanisms of entry are not clear. If AZT is administered to the mother, a clinically efficient dose is generated within the placental cells, and therefore it may possibly be a point in the pathway at which infection is prevented. Further investigations must be ideally performed; if this was an effective preventative measure then there may be evidence of infected placentae and infection-free fetuses. Alternatively, virus levels could be monitored within the placenta throughout pregnancy and compared with the maternal virus levels to see whether these are concordant, and where the AZT is having its optimal effect. These may not be ethically possible to determine, but they are some of the questions that should be considered in order to gain further information on this subject.

\section{In the Fetus}

It is known from evidence detecting HIV in fetal tissue as early as the eighth week of gestation and the second trimester, as well as HIV-antigens in the thymic T4 cells of third trimester fetuses $(26,39)$, that a significant proportion of materno-fetal infection is intrauterine. The important point to draw from this is that since HIV can infect the fetus, AZT may perhaps be effective in preventing infection at this stage.

AZT crosses the placenta and enters the fetal circulation, rapidly achieving levels comparable to those in maternal blood (41). Thus, prevention and/or treatment of fetal infection may be possible through indirect therapy to the fetus via transplacental passage of maternal AZT. If a considerable portion of transmission occurs at the time of delivery, then the presence of systemic AZT levels in the newborn might achieve significant prophylaxis. Rapid transplacental transport of a loading dose, followed by continuous 
infusion during labor, provides substantial AZT concentrations in the newborn. Administration of postpartum AZT constitutes post-exposure prophylaxis. However, from a fetal safety viewpoint, the risks of intervention must be less than the risk of vertical transmission, and also evaluated in light of concurrent maternal risks and clinical conditions.

In order to examine this possible mechanism of AZT, and in view of the scarcity of material regarding fetuses and the newborn, it may be necessary to consider evidence of the prophylactic effect of AZT in adults. This has received attention due to the use of AZT to prevent infection in health care workers exposed to HIV; for example, a French case-control study of health care workers with percutaneous exposure to HIV suggests that AZT use might be protective as a post-exposure measure (42).

Other experiments show similar results, including a study on macaques using Simian Immunodeficiency Virus (SIV), inoculated seven days after AZT therapy was begun. There was a significant delay $(p=0.011)$ in the appearance of cell-associated and cell-free viremia in the AZT treated monkeys as compared to the untreated; however, no protection from SIV infection was observed (43). Overall there is no clear evidence that AZT prevents seroconversion or infection after exposure to HIV.

All of the above studies were carried out on adult humans or animals. Understandably, there has been little experimentation on newborns. One report (44) of SIV infection in newborn Rhesus monkeys indicates that prevention may be observed in such situations, and if transmission does occur, an attenuated infection may be seen. In the nine newborn monkeys inoculated with SIV and then given immediate oral AZT, infection was prevented in one animal and decreased viral load and delayed disease progression were observed in the eight others, who became infected despite AZT. As this study involved only a small sample size and did not strictly test HIV infection as a result of vertical transmission, it cannot form the basis of any definitive conclusions, although it does offer a small chance that prevention of infection is possible.

Information regarding the distribution of AZT in the fetus is virtually non-existent. There is a possibility that AZT offers protection against infection or against viral replication, either during gestation or after exposure at birth and in the postpartum period. Therapeutic concentrations of the drug are present, and its virustatic qualities are known. However, there is little significant evidence of effective prophylaxis, even in adults. If an AZT effect in the fetus is the mechanism by which AZT reduces the risk of materno-infant transmission, then the next step would be to look for infected placentas and seronegative fetuses and newborns, or perhaps the AZT therapy has merely delayed the onset of viremia and HIV symptoms, and these become apparent later. The idea of AZT prophylaxis is important, as it also has repercussions for others who are exposed to HIV in isolated incidents. However, experiments in fetuses and newborns are profoundly difficult; thus, future investigative procedures must use experimental models and extrapolate to humans.

\section{CONCLUSION}

As presented in this review, AZT may be acting to prevent vertical HIV transmission within the mother (primarily by reduction of the maternal viral burden), within the placenta (by prevention of either placental infection or fetal exposure) and within the fetus (by some prophylactic effect). There is little direct evidence for any of these, and although the proposed mechanisms have been presented distinctly, it is important to emphasize that they are in no way mutually exclusive and may even work concurrently.

The mechanism of AZT is significant, because it provides the key to apply the treatment regimen, or variations on it, to different clinical situations and individuals with an understanding and insight into how the results are achieved. Throughout this review, references have been made to areas of research requiring greater attention. The major difficulties with this line of work include a lack of a good animal or experimental models, and the inability to isolate each stage to observe the effects of AZT, e.g., the different stages of pregnancy, or the different compartments of mother, placenta and fetus.

The possibility that AZT therapy may be effective in preventing vertical transmission, could bring calls for mandatory unblinded testing of newborns and even of all pregnant women. This also raises the critical problem of the cost of such services to the individual and to health authorities as well as ethical dilemmas regarding an individual's rights of self-determination.

The advent of combination therapy, utilizing an ever broadening pharmaceutical armamentarium, provides further hope for HIV-positive mothers in the Western world. Techniques used to treat individuals at risk of developing resistance to AZT may now have relevance to mothers previously treated with AZT or at later stages of disease. Despite the importance of this breakthrough, it is likely to have little or no effect on women in developing nations where the toll of pediatric AIDS is most severe, an estimated 95\% of the world's HIV-infected women $(45,46)$. The ACTG076 protocol of testing, counselling, acceptance of medication, intravenous infusion of AZT during labor and ban on breast feeding, is almost impossible to implement in areas such as Malawi, for example, where reports (47) from the Queen Elizabeth Central Hospital show that 
$58 \%$ of women give birth within three hours of arrival on their first visit, and $80 \%$ within seven hours, having no time to even determine the woman's HIV status. There is a great need for an economically and logistically sustainable measure against vertical transmission in these areas, and the ACTG076 regimen is unfeasible.

In response to this situation, several studies were initiated world-wide in an attempt to find an alternative to AZT therapy or a more workable protocol, including using microbiocides during labor, intravenous immune globulin (IVIG), HIV Hyperimmune globulin (HIVIG), and vitamin A (3). The most exciting of these trials published its preliminary results in March 1998 (48). This Thai study involved a sample size of 392 women randomized to receive oral AZT or placebo from 36 weeks gestation and throughout labor until delivery. Infants were not treated, but were also not breastfed. There was no limitation as the maternal CD4 count. The results using a Kaplan-Meier analysis showed estimated HIV transmission risks: $9.2 \%$ in the AZT group (95\% confidence limit, $5.0 \%$ to $13.5 \%$ ) and $18.6 \%$ in the placebo group (95\% confidence limit, $13 \%$ to $24 \%$ ). This represents an absolute risk reduction of $9.4 \%$ and a relative risk reduction of $51 \%(p=0.008)$. These results can be interpreted as only needing to treat 11 women to prevent one more infant infection, and so suggest that short term oral AZT therapy may be a viable option for developing countries.

In conclusion, although questions remain and much work needs to be done, the findings of the ACTG076 trial allow us a glimmer of hope of preventing a terrible and devastating disease from infecting children. The challenge is now to implement these findings in an effective, ethical and practical way so as to fulfill the potential of the trial for maximum clinical success.

\section{ACKNOWLEDGMENTS}

The author wishes to thank Dr. Graham Burton (Department of Anatomy, Cambridge University, UK) for his patient supervision of this project and for his support over the past years, and Dr. O'Shea (St. Thomas' Hospital, London, UK) for her help and advice.

\section{REFERENCES}

1. Gayle H. Epidemiology of HIV infection, with a focus on mother-to-child transmission and among drug users. Current Opinions in Infectious Diseases 6: 200-204; 1993.

2. Banatvala JE, O'Shea S, Newell ML, et al. Studies on HIV infected women and their infants. Research fund proposal; 1995.

3. Fowler MG, Rogers MF. Overview of perinatal HIV infection. Journal of Nutrition 126: 2602S - 2607S; 1996.

4. Rogers MF, Jaffe HW. Reducing the risk of maternal-infant transmission of HIV: a door is opened. New England Journal Of Medicine 331: 1222-1223; 1994.
5. Thisyakorn U, Ruxrungtham K, Phanuphak P. HIV and AIDS Current Trends 4: 1-3; 1998.

6. Gerold M., Adler R. Manifestations of pediatric AIDS: proposed mechanisms for transmission. Medical Hypotheses 37: 205-212; 1992.

7. Sager P. Conference report: workshop on the role of the placenta in HIV infection and therapy - Nantucket, Massachusetts, September 1990. Placenta 12: 669-672; 1991.

8. Newell ML. European collaborative study - risk factors for vertical transmission. European Collaborative Study Conference Report; 1992: 6-13.

9. Connor EM, Sperling RS, Gelber R. et al. Reduction of maternal-infant transmission of the human immunodeficiency virus type 1 with zidovudine treatment. New England Journal of Medicine 331: 1173-1180; 1994.

10. Clinical Alert. Important therapeutic information on the benefit of zidovudine for the prevention of the transmission of HIV from mother to infant. ACTG Site Instruction Package; 1994.

11. Connor EM, Mofenson LM. Zidovudine for the reduction treatment of perinatal human immunodeficiency virus transmission: pediatric AIDS clinical trials group 076 - results and treatment recommendations. Pediatric Infectious Disease Journal 14: 125-131; 1995.

12. Boyer PJ, Dillon M, Navaie M, et al. Factors predictive of maternal-fetal transmission of HIV-1. JAMA 271: 1925-1930; 1994.

13. Matheson PB, Abrams EJ, Thomas PJ, et al. Efficacy of antenatal zidovudine in reducing perinatal transmission of human immunodeficiency virus type 1. Journal of Infectious Diseases 172: 353-358; 1995.

14. Cooper E, Diaz D, Pitt J, et al. Impact of ACTG076: use of zidovudine (ZDV) during pregnancy and changes in the rate of HIV vertical transmission. 3rd Conference of Retro and Opportunistic Infectection 57; 1996.

15. Whiting RB. The response of state health departments and national associations to the results of ACTG 076. International Conference on AIDS 11: 410; 1996.

16. Simpson BJ, Shapiro ED, Andiman WA, et al. Reduction in the risk of vertical transmission of HIV-1 associated with treatment of pregnant women with orally administered zidovudine alone. Journal of Acquired Immune Deficiency Syndromes and Human Retrovirology 14: 145-152; 1997.

17. Martinez-Tejada B. Zidovudine prophylaxis for the prevention of HIV vertical transmission in the hospital clinic of Barcelona. International Conference on AIDS 11: 77; 1996.

18. Cahn P. Zidovudine (ZDV) for prophylaxis of perinatal transmission starting on week 24. 4th Conference on Retroviruses and Opportunistic Infections 159; 1997.

19. Watts DH, Brown ZA, Tartaglione T, et al. Pharmacokinetic disposition of zidovudine during pregnancy. Journal of Infectious Diseases 163: 226-232; 1991.

20. O'Sullivan MJ, Boyer PJ. The pharmacokinetics and safety of zidovudine in the third trimester of pregnancy for women infected with the Human Immunodeficiency Virus and their infants - Phase I ACTG 082. American Journal of Obstetrics and Gynaecology 168: 1510-1516; 1993.

21. Liebes L, Mendoza S, Wilson D, et al. Transfer of zidovudine (AZT) by the human placenta. Journal of Infectious Diseases 161: 203-207; 1990.

22. Liebes L, Mendoza S, Lee JD, et al. Further observations on zidovudine transfer and metabolism by the human placenta. AIDS 7: 590-592; 1993.

23. Pons JC, Tabuset AM, Singlas E, et al. Placental passage of azathiothymidine (AZT) during the second trimester of pregnancy: study by direct fetal blood sampling. European 
Journal of Obstetrics and Gynaecology and Reproductive Biology 40: 229-31; 1991.

24. Gillet JY, Garraffo R, Abrar D, et al. Fetoplacental passage of zidovudine. Lancet (2): 269-270; 1989.

25. Bui T, Bark D, Perkins M, et al. Effect of zidovudine on human placental trophoblast and Hofbauer cell functions. Journal of AIDS 6: 120-126; 1993.

26. Mofenson LM. Timing and mechanisms of HIV vertical transmission: implications for prevention. Archives of STD/HIV Research 7: 207-211; 1993.

27. European collaborative study. Risk factors for mother to child transmission. Lancet 339: 1007-1012; 1992.

28. The HIV Infection in Newborns French Collaborative Study Group. Comparison of vertical HIV type 2 and HIV type 1 transmission in the French prospective cohort. Pediatric Infectious Diseases 13: 502-506; 1994.

29. Dunn DT, Newell ML, Ades AE, et al. Risk of HIV type 1 transmission through breastfeeding. Lancet 340: 585-588; 1992.

30. Minkoff H, Burns D, Landesman SH. The relationship of the duration of ruptured membranes to vertical transmission of HIV. American Journal of Obstetrics and Gynaecology 173: 585-589; 1995.

31. Scarlatti G, Albert J, Rossi P, et al. Comparison of variable region 3 sequences of HIV type 1 from infected children with the RNA and DNA sequences of the virus populations of their mothers. Proceedings of the National Academy of Science (USA) 90: 1721-1725; 1993

32. Sperling RS, Shapiro D, Coombs RW, et al. Maternal viral load, zidovudine treatment and the risk of transmission of HIV type 1 from mother to infant. New England Journal of Medicine 335: 1621-1629; 1996.

33. O'Shea S, Newell ML, Dunn DT, et al. Maternal viral load, CD4 cell count and vertical transmission of HIV-1. Journal of Medical Virology 54: 113-117; 1998.

34. Minkoff $\mathrm{H}$, Mofenson LM. The role of obstetric interventions in the prevention of pediatric HIV infection. American Journal of Obstetrics and Gynaecology 171: 1167-1173; 1994.

35. Ho DD, Moudgil T, Alam M, et al. Quantitation of human immunodeficiency virus type 1 in the blood of infected persons. New England Journal of Medicine 321: 1621-1625; 1989.

36. Henin Y, Mandelbrot L, Henrion R, et al. Virus excretion in the cervicovaginal secretions of pregnant and non-pregnant HIVinfected women. Journal of AIDS 6: 72-75; 1993.
37. Burton GJ, O'Shea S, Rostron T, et al. Physical breaks in the placental trophoblastic surface: significance in vertical transmission. AIDS 10: 1294-1296; 1996.

38. Kesson AM, Fear WR, Williams L, et al. HIV Infection of Placental Macrophages: their potential role in vertical transmission. Journal of Leukocyte Biology 56: 241-246; 1994.

39. Lewis SH, Reynolds-Kohler C, Fox ME, et al. HIV-1 in trophoblastic and villous Hofbauer cells, and haematological precursors in 8-week foetuses. Lancet 335: 565-568; 1990.

40. Furman PA, Fyfe JA, St. Clair MH, et al. Phosphorylation of 3'azido-3'-deoxythymidine and selective interaction of the 5'triphosphate with HIV Reverse Transcriptase. Proceedings of the National Academy of Science (USA) 83: 8333-8337; 1986.

41. Kumar RM, Hughes PF, Khurrana A, et al. Zidovudine use in pregnancy: a report of 104 cases and the occurrence of birth defects. Journal of AIDS 7: 1034-1039; 1994.

42. Case control study of HIV seroconversion in health care workers after percutaneous exposure to HIV infected blood France, United Kingdom, and United States, Jan 1988 - August 1994. Morbidity Mortality Weekly Report 44: 929-933; 1995.

43. Le Grand R, Clayette P, Noack O, et al. An animal model for antilentiviral therapy: effect of zidovudine on viral load during acute infection after exposure of macaques to simian immunodeficiency virus. AIDS Research and Human Retroviruses 10: 1279-1287; 1994.

44. Van Rompay KKA, Otsyola MG, Marthas ML, et al. Immediate zidovudine treatment protects simian immunodeficiency virusinfected newborn macaques against rapid onset of AIDS. Antimicrobial Agents and Chemotherapy 39: 125-131; 1995.

45. Bayer R. Ethical challenges posed by Zidovudine treatment to reduce vertical transmission of HIV. New England Journal of Medicine 331: 1223-1225; 1994.

46. Meda N, Msellati P, Welflens-Ekra C, et al. The reduction of mother-child transmission of HIV infection in developing countries: potential intervention strategies, obstacles to implementation and perspectives. Sante 7:115-125; 1997.

47. Hoffman CA. Ethical issues in the use of zidovudine to reduce vertical transmission of HIV. New England Journal Of Medicine 332: 891-892; 1995.

48. Administration of zidovudine during late pregnancy and delivery to prevent perinatal HIV transmission - Thailand, 19961998. Morbidity Mortality Weekly Report 47: 151-154; 1998.

V. Bhargavi Rao is currently a final year medical student at Oxford University (University College, Oxford, UK). She performed her preclinical studies and Part II Tripos at Cambridge University (St. John's College, Cambridge, U.K.; 19931996), gaining a First Class BA Honours (Cantab) degree. Her research on the use of AZT to reduce the risk of vertical transmission was conducted in her final year in Cambridge, as part of a Part II in Anatomy B "Disease, Society, and Sexuality". Parts of this research were presented at the 80th anniversary conference of the Women's Medical Federation (MWF) entitled "Preventable Disasters in Women's Health", in November 1997. 